\title{
Which way is which? Examining symbolic control of attention with compound arrow cues
}

\author{
Mark Mills $^{1,2} \cdot$ Michael D. Dodd ${ }^{1}$
}

Published online: 1 June 2016

(C) The Psychonomic Society, Inc. 2016

\begin{abstract}
Spatial symbols can generate attentional biases toward peripheral locations compatible with the symbol's meaning. An important question concerns how one symbol is selected when competing symbols are present. Studies examining this issue for spatially distinct symbols have suggested that selection depends on the task goals. In the present study, we examined whether the influence of competing symbolic stimuli (arrows) at different levels of structure on attentional control also depends on the task goals. Participants made simple detection responses to a peripheral target preceded by a spatially uninformative compound arrow (global arrow composed of local arrows). In addition, participants were required to perform a secondary task in which they matched the orientation of the global arrow (global task) or the location of a uniquely colored local arrow (local task) to a test display presented immediately following a detection response. When the global and local arrows pointed at opposite locations, a local cueing effect emerged in the local task, and a global cueing effect in the global task, indicating that the task goals influenced the selection of the level of structure. However, when the local level was spatially neutral (global arrow, local rectangles), a cueing effect was observed independent of task, and when the global level was spatially neutral (global rectangle, local arrows), a cueing effect was observed in the local task only, suggesting that global processing was obligatory and local processing optional. These findings suggest that
\end{abstract}

Mark Mills

mark.mills2@huskers.unl.edu

University of Nebraska, Lincoln, NE, USA

2 Department of Psychology, University of Nebraska-Lincoln, 238 Burnett Hall, Lincoln, NE 68588, USA attentional effects triggered by the global level are more strongly reflexive than those triggered by the local level.

Keywords Attention $\cdot$ Symbolic control $\cdot$ Contingent capture $\cdot$ Compound cue $\cdot$ Global/local

Everyday visual environments generally contain more information than can be processed within a glance. As such, goaldirected perception and action depend on mechanisms of selective visual attention for prioritizing an endless stream of sensory input, and so giving more weight to those objects, locations, or events that require immediate or sustained processing. To understand how attention is prioritized and allocated to visual stimuli, a useful distinction is that between bottom-up (reflexive) and top-down (volitional) attentional control, with the former driven by the physical characteristics of a stimulus and the latter by the current goals of the observer (Corbetta \& Shulman, 2002; Desimone \& Duncan, 1995; Egeth \& Yantis, 1997; Itti \& Koch, 2000; Jonides, 1981; Posner, 1980; see Yantis, 2000, for a review). For example, an abrupt visual onset may on the one hand capture attention in a purely stimulus-driven manner (Enns, Austen, Di Lollo, Rauschenberger, \& Yantis, 2001; Remington, Johnston, \& Yantis, 1986; Theeuwes, 1990, 1994; Yantis \& Jonides, 1984). On the other hand, capture may be contingent on the top-down attentional set of an observer, such that the onset captures attention only if it shares a feature with an item in that set (Folk \& Remington, 1998; Folk, Remington, \& Johnston, 1992).

Outside of the traditional top-down/bottom-up dichotomy, considerable evidence exists indicating that various kinds of symbolic information presented at fixation produce unintentional shifts of attention to peripheral locations that are compatible with the meaning of the symbol. For example, 
Hommel, Pratt, Colzato, and Godijn (2001) presented a centrally located directional arrow (e.g., <, >) or word (e.g., left, right), which was followed by a peripheral target requiring a detection response. Targets were detected more quickly when they appeared at the location indicated by the arrow or word (valid condition) than at another location (invalid condition). Importantly, this occurred even though these symbols were entirely irrelevant to the detection task, and observers were explicitly told that the symbols did not predict the location of the upcoming target (see also Ristic \& Kingstone, 2006, 2012; Ristic, Landry, \& Kingstone, 2012). Similar findings have been observed for temporal words (e.g., tomorrow, yesterday; Weger \& Pratt, 2008), words relating to concrete concepts (e.g., head, foot; Estes, Verges, \& Barsalou, 2008), words relating to abstract concepts (e.g., god, devil; Chasteen, Burdzy, \& Pratt, 2010), pictures relating to abstract concepts (e.g., liberal, conservative; Mills, Smith, Hibbing, \& Dodd, 2015), numbers (Fischer, Castel, Dodd, \& Pratt, 2003), and letters (Dodd, Van der Stigchel, Leghari, Fung, \& Kingstone, 2008). Taken together, these findings indicate that a broad range of visual symbols can produce unintentional shifts of attention (but see Fattorini, Pinto, Rotondaro, \& Doricchi, 2015)

Not all visual symbols capable of producing unintentional shifts of attention are likely to do so in all situations, however. For example, observers are sometimes faced with scenes in which one symbol is nested within another, potentially conflicting symbol (e.g., eyes looking one way nested within a head looking another way). Assuming that shifts of attention cannot be made in two or more directions simultaneously, an important question is which level might be selected (e.g., global head or local eyes) to control the allocation of attention? In the present study, we examined this issue using compound stimuli for which observers should have no a priori basis for selecting one level over the other (a global arrow composed of local arrows). One possibility is that the level that is relevant to the current task goal would be selected, and the arrow at this task-relevant level would then produce an unintentional shift of attention. Evidence for this hypothesis has come from research on the role of control settings in attentional capture. The framework of attentional control settings (Folk et al., 1992) proposes that the processing of a stimulus feature is contingent on the task goal at hand, such that only stimuli possessing a task-relevant feature can pass through a perceptual filter and enter working memory. For instance, Pratt and Hommel (2003) examined how one symbol is selected to control the allocation of attention when several symbols appear in the visual field. They found that in a field of spatially distinct arrows, the arrow most likely to affect attentional control was the one that possessed a task-relevant feature of an expected target. This suggests that the unintentional effects of arrows depend on the cognitively represented task goal. Thus, the control-setting account predicts that only the arrow that shares a critical feature specified by top-down control settings will be selected to control the allocation of attention.

Another possibility is that the global level would be selected first, regardless of task, and that this global arrow would then produce an unintentional shift of attention. Evidence for this hypothesis has come from research on the role of global perceptual precedence in the perception of hierarchical structure. Global precedence (Navon, 1977, 2003) proposes that perceptual processing is predisposed to favor the processing of "clusters," such that the global form of a hierarchical structure is registered earlier than its local constituents, resulting in greater availability of the global than of the local percept. As such, processing of global information is assumed to be obligatory, whereas the processing of local information is optional. Navon (1977) tested this notion in experiments involving compound stimuli - stimuli with hierarchical levels of structure (e.g., a large, global F constructed from small, local Hs). Observers were presented with these stimuli and instructed to identify the letter at either the global or the local level. Importantly, the relation between the levels was either consistent (global F, local Fs) or inconsistent (global F, local Hs). The critical findings were that global were identified faster than local letters (global advantage) and that processing of the local level was slowed to a greater extent than processing of the global level when the global and local object identities were inconsistent (global interference). Such findings indicate that global properties can be extracted rapidly, suggesting that the potency of an arrow to evoke an unintentional shift of attention may depend on its globality (i.e., its relative position in hierarchical space).

Navon's (1977, 2003) global-precedence hypothesis assumes that the availability of the global level is constant and, therefore, that findings of global advantage should not vary over time. Subsequent work, however, has suggested that this assumption may be too strong. In particular, there is evidence that the availability of different levels of structure of hierarchical patterns changes over time. Kimchi (1998) examined the microgenesis of the perceptual organization of hierarchical stimuli using a primed-matching task. Observers were presented with a prime (either a few- or a many-element hierarchical pattern), followed by a pair of test figures to be matched for identity. In the element similarity test pair condition, the test figures were similar to the prime in their elements, but different in their global configuration. In the configuration similarity test pair condition, the test figures were similar to the prime in their global configuration, but different in their elements. By varying the duration of the prime and constructing test figures that were similar to its different aspects, this paradigm allowed changes in observers' implicit perceptual representations over time to be measured. With few-element patterns, the elements were primed at brief exposures, whereas the configuration was primed at longer exposures. In contrast, 
with many-element patterns, the configuration was primed at brief exposures, whereas the elements were primed at longer exposures. Similar findings have been obtained with compound arrows. Mills and Dodd (2014) presented a spatially uninformative and task-irrelevant compound arrow (a large, global arrow constructed from smaller, local arrows) at fixation, which was followed by a peripheral target requiring a simple detection response. The directions of the global and local arrows were either consistent (same direction) or inconsistent (different directions). The critical manipulation was stimulus onset asynchrony (SOA). The rationale of the paradigm is that the priming potency of a level should vary with SOA in correspondence with how early that level is processed (Navon, 1991). The results indicated that inconsistent arrows produced a global cueing effect (target detection was faster when the global rather than the local level pointed toward the target) at short SOAs, consistent with a global advantage. At long SOAs, however, a local cueing effect was observed (target detection was faster when the local rather than the global level pointed toward the target), indicative of a local advantage. Taken together, these findings suggest that that the unintentional effects of arrows may depend not only on the globality of an arrow, but also on its availability over time.

In sum, previous work suggested that the potency of symbols to evoke unintentional shifts of attention may vary with their globality and the availability of that percept over time, or with their relevance to the current task. Accordingly, in the present study we investigated whether the relative impacts of competing symbolic stimuli on attentional control depend on top-down selection processes. In particular, we used the compound-arrow cueing task introduced by Mills and Dodd (2014) to examine the effects of a top-down orienting task on the selection of information at the global and local levels of a compound arrow cue. Each trial consisted of two major events (Fig. 1). First, a spatially uninformative compound arrow cue was presented at fixation. The cues were either consistent (global and local arrows pointing in the same direction), inconsistent (global and local arrows pointing in opposite directions), local-neutral (a global arrow composed of local rectangles), or global-neutral (a global rectangle composed of local arrows). Second, after a variable delay, a target was presented to the left or right of fixation requiring a simple detection response. Participants are instructed that their primary task was target detection, but that they should also perform one of two secondary tasks. In the global orienting task, the arrow cues were oriented either perfectly in line with or slightly above or below the horizontal midline, and participants were to remember the orientation of the big arrow. In the local orienting task, one of the local arrows was a color singleton, and participants were to remember its location. Following the detection response, a test display was shown presenting two compound arrows, and participants were to select the one that matched what they had just seen.

For consistent cues, the perceptual-precedence and control-setting accounts both predict the presence of a
Fig. 1 Compound arrow cues and validity conditions, as well as example trial sequences in Experiment 1. The left panel shows example cue stimuli used for the global orienting task, in which the orientation of the global arrow was task-relevant. The right panel shows example cue stimuli used for the local orienting task, in which the location of a uniquely colored local arrow/ rectangle was task-relevant. Both sets of cue stimuli were presented in color during the experiment, with local arrows colored red (represented here as gray). In the local orienting task, the color singleton was green (represented here as black)

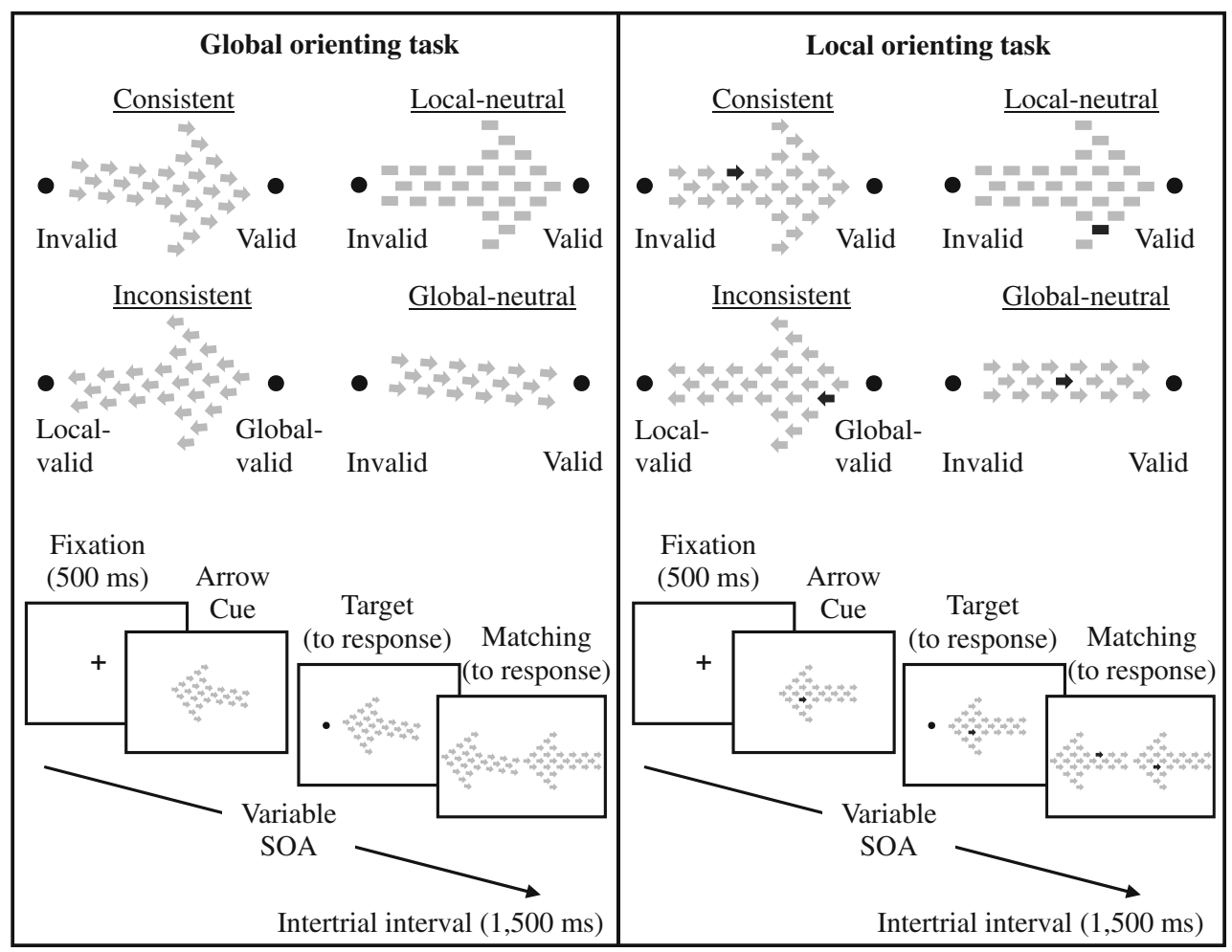


spatial cueing effect. For inconsistent cues, however, the two accounts make different predictions. According to the perceptual-precedence account, information at the global level is available earlier than information at the local lev$\mathrm{el}$, and therefore receives prioritized processing. For inconsistent cues, then, this account predicts that the global level would be selected first, regardless of the orienting task, and that the arrow at this level would then produce an unintentional shift of attention. If a target did not appear at the location indicated by the global arrow, attention might be reoriented back to center and a local arrow could be selected, provided that the local level was taskrelevant. Thus, the perceptual-precedence account predicts an early, task-independent global cueing effect, which might later give way to a local cueing effect when the local level was task-relevant and the levels conflicted. According to the control-setting account, in contrast, only arrows possessing a task-relevant feature would enter working memory and compete for attentional control. Therefore, for inconsistent cues, this account predicts a task-compatible level-specific cueing effect, such that a global cueing effect should be observed in the global orienting task, whereas a local cueing effect should be observed in the local orienting task.

The two accounts also differ in their predictions for local-neutral cues. The perceptual-precedence account predicts that because the global level is always prioritized, it would be selected regardless of orienting task, and the arrow at this level would produce an unintentional shift of attention. Furthermore, because there was no conflict between levels, the cueing effect should not vary with SOA. In contrast, the control-setting account predicts that the global level would be selected only when it was taskrelevant. Thus, the global cueing effects triggered by local-neutral cues should be observed in the global but not in the local orienting task. The two accounts make similar predictions for global-neutral cues, albeit for different reasons. The perceptual-precedence account predicts that because processing of the global level is obligatory, whereas processing of the local level is optional, the local level should be processed only when it is taskrelevant. Therefore, this account predicts a local cueing effect in the local orienting task (given that the local level is task-relevant and contains arrows), but not in the global orienting task (given that the global level does not contain an arrow). Furthermore, because here there is no conflict between levels, the local cueing effects triggered by global-neutral cues should not vary with SOA. The control-setting account predicts that the local level would be selected only when it was task-relevant. The local cueing effects triggered by global-neutral cues, therefore, should be observed in the local but not in the global orienting task.

\section{Experiment 1}

\section{Method}

Participants Thirty-eight undergraduates from the University of Nebraska-Lincoln participated in exchange for course credit. All of the participants had normal or corrected-tonormal vision, were naive to the purpose of the experiment, and were informed of their rights of participation according to the University of Nebraska-Lincoln institutional review board.

Stimuli The compound arrow cues are shown in Fig. 1. The consistent and inconsistent cues were structured such that 26 local arrows (each subtending $0.625^{\circ} \times 0.50^{\circ}$ visual angle) yielded a single global arrow $\left(7.5^{\circ} \times 5.0^{\circ}\right)$. The local-neutral cues were 26 local rectangles (each subtending $0.625^{\circ} \times$ $\left.0.50^{\circ}\right)$ arranged to form a global arrow $\left(7.5^{\circ} \times 5.0^{\circ}\right)$. The global-neutral cues were 17 local arrows (each subtending $0.625^{\circ} \times 0.50^{\circ}$ ) arranged to form a single global rectangle $\left(7.0^{\circ} \times 3.0^{\circ}\right)$. In the local orienting task, the local arrows/ rectangles were filled with red except for a single arrow/ rectangle that was filled with green. In the global orienting task, the local arrows/rectangles were filled with red, and the global arrow/rectangle was oriented about the horizontal midline $-10^{\circ}, 0^{\circ}$, or $10^{\circ}$. The cues in both tasks were presented on a white background.

Procedure An example trial sequence for each orienting task is shown in Fig. 1. Each trial began with a central fixation point (a black "+" subtending $1^{\circ}$ of visual angle) and was replaced by a compound arrow cue after $500 \mathrm{~ms}$, which remained onscreen until a response was made on the primary detection task. After a variable SOA $(250,500$, or $750 \mathrm{~ms})$, a target (a black circle subtending $1^{\circ}$ of visual angle) was presented, requiring simple target detection. The cue direction and target location were presented with equal probability leftward ("<") or rightward (">") and to the left or right of fixation, respectively. The combination of these factors indexed each cue's validity state. Thus, the cues were either consistent (global and local arrows pointing in the same direction), inconsistent (global and local arrows pointing in opposite directions), local-neutral (global arrow only), or global-neutral (local arrows only), and either valid (pointing toward target location) or invalid (pointing away from target location). Because the inconsistent cues were technically always valid, given that either the global or the local level would always point at the target location, we refer to the conditions of its validity state as global-valid (global level pointing at target location) and local-valid (local level pointing at target location). Accordingly, for inconsistent cues, the usual indicator of attentional orienting (the response time [RT] advantage for validly vs. invalidly cued target locations) now served as an 
Table 1 Mean response times (RTs), in milliseconds, and mean probabilities of error, $p$ (error), in each cue by stimulus onset asynchrony condition in Experiment 1

\begin{tabular}{|c|c|c|c|c|c|c|c|c|}
\hline \multirow[t]{3}{*}{ Condition } & \multicolumn{4}{|c|}{ Local Orienting Task } & \multicolumn{4}{|c|}{ Global Orienting Task } \\
\hline & \multicolumn{2}{|c|}{ Valid $^{\mathrm{GV}}$} & \multicolumn{2}{|c|}{ Invalid $^{\mathrm{LV}}$} & \multicolumn{2}{|c|}{ Valid $^{\mathrm{GV}}$} & \multicolumn{2}{|c|}{ Invalid $^{\mathrm{LV}}$} \\
\hline & $M$ & $S E$ & $M$ & $S E$ & $M$ & $S E$ & $M$ & $S E$ \\
\hline \multicolumn{9}{|l|}{$\mathrm{RT}(\mathrm{ms})$} \\
\hline \multicolumn{9}{|c|}{ Consistent } \\
\hline 250 & 454 & 14 & 469 & 14 & 436 & 14 & 452 & 14 \\
\hline 500 & 386 & 14 & 405 & 14 & 374 & 14 & 391 & 14 \\
\hline 750 & 376 & 14 & 375 & 14 & 367 & 14 & 393 & 14 \\
\hline \multicolumn{9}{|c|}{ Inconsistent } \\
\hline 250 & 489 & 15 & 475 & 15 & 448 & 15 & 454 & 15 \\
\hline 500 & 408 & 15 & 395 & 15 & 378 & 15 & 395 & 15 \\
\hline 750 & 381 & 15 & 378 & 15 & 366 & 15 & 393 & 15 \\
\hline \multicolumn{9}{|c|}{ Local-neutral } \\
\hline 250 & 403 & 12 & 426 & 12 & 394 & 12 & 406 & 12 \\
\hline 500 & 338 & 12 & 352 & 12 & 333 & 12 & 345 & 12 \\
\hline 750 & 322 & 12 & 332 & 12 & 326 & 13 & 327 & 12 \\
\hline \multicolumn{9}{|c|}{ Global-neutral } \\
\hline 250 & 409 & 12 & 412 & 12 & 407 & 12 & 411 & 12 \\
\hline 500 & 330 & 12 & 350 & 12 & 334 & 12 & 337 & 12 \\
\hline 750 & 319 & 12 & 334 & 12 & 331 & 12 & 332 & 12 \\
\hline \multicolumn{9}{|l|}{$p$ (error) } \\
\hline \multicolumn{9}{|c|}{ Consistent } \\
\hline 250 & .184 & .040 & .245 & .049 & .217 & .046 & .220 & .046 \\
\hline 500 & .217 & .046 & .186 & .041 & .178 & .040 & .208 & .045 \\
\hline 750 & .252 & .051 & .185 & .041 & .158 & .037 & .202 & .044 \\
\hline \multicolumn{9}{|c|}{ Inconsistent } \\
\hline 250 & .268 & .053 & .249 & .050 & .167 & .038 & .202 & .043 \\
\hline 500 & .203 & .044 & .162 & .037 & .191 & .042 & .168 & .038 \\
\hline 750 & .223 & .047 & .175 & .039 & .217 & .046 & .212 & .045 \\
\hline \multicolumn{9}{|c|}{ Local-neutral } \\
\hline 250 & .163 & .041 & .182 & .045 & .173 & .043 & .187 & .045 \\
\hline 500 & .178 & .045 & .184 & .045 & .181 & .045 & .208 & .049 \\
\hline 750 & .166 & .043 & .152 & .040 & .171 & .044 & .179 & .045 \\
\hline \multicolumn{9}{|c|}{ Global-neutral } \\
\hline 250 & .143 & .037 & .185 & .045 & .139 & .037 & .163 & .041 \\
\hline 500 & .135 & .036 & .166 & .042 & .125 & .034 & .186 & .046 \\
\hline 750 & .165 & .043 & .134 & .036 & .176 & .045 & .174 & .044 \\
\hline
\end{tabular}

$\mathrm{GV}=$ global valid, $\mathrm{LV}=$ local valid .

indicator of level-specific attentional orienting. Participants were instructed to press the spacebar as quickly as possible once the target appeared, and to maintain central fixation throughout. Moreover, participants were informed that the central arrows did not predict the target location and, therefore, were irrelevant to the detection task.

To induce voluntary selection of the global or local arrows, a secondary task was used to orient attention toward either the global or the local level. Participants were told that their primary task was target detection but that they should also try to perform a secondary task in preparation for a test given at the end of each trial. The secondary task was a matching task in which participants matched either the orientation of the global arrow (global orienting task) or the location of a uniquely colored local arrow (local orienting task) to one of two alternatives in a test display. The test displays were presented immediately following a detection response and remained present until a discrimination response. The intertrial interval was $1,500 \mathrm{~ms}$. Participants were seated $48 \mathrm{~cm}$ from the monitor and used the keyboard to make detection responses (spacebar) and discrimination responses (the $a$ or $s$ key for a left or a right response, with the mapping counterbalanced across participants). Testing took place on a Pentium IV computer with a 17-in. monitor in a room equipped with soft lighting and sound attenuation. The experimental sessions took place individually and lasted $\sim 30 \mathrm{~min}$.

Design Participants saw each cue (consistent, inconsistent, local-neutral, or global-neutral) at each SOA $(250,500$, or $750 \mathrm{~ms}$ ) and validity state (for consistent and neutral cues, valid or invalid; for inconsistent cues, global-valid or localvalid) for each orienting task (global or local). These 48 
conditions were repeated ten times, for a total of 480 trials. Cue type was blocked, such that consistent and inconsistent cues were presented in one block, and local-neutral and global-neutral cues were presented in another block. The block order was fixed across participants, with the local-neutral/global-neutral block always performed last. Within each cue block, orienting task was also blocked, with the task order counterbalanced across participants.

\section{Results and discussion}

Accuracy on the secondary matching task We first report the accuracy data on the secondary matching task. Because accuracy was binary (correct or incorrect), we modeled the log-odds of the probability of an errant matching response (Hoffman, 2014). The model included a random intercept for subjects and by-subject random slopes for withinsubjects manipulations (Barr, Levy, Scheepers, \& Tily, 2013). The bottom half of Table 1 shows the mean probabilities of an error in each condition. The overall probability of an error was .18. Though this is somewhat high for relatively easy tasks, it was expected because participants were instructed to prioritize speed on the primary target detection task. We found a main effect of cue type, $F(3,91.5)=8.94, p<$ .001 , in which the mean probabilities of error were greater with consistent $(M=.203, S E=.040)$ and inconsistent $(M=$ $.201, S E=.039)$ cues than with local-neutral $(M=.177, S E=$ $.036)$ and global-neutral $(M=.157, S E=.033)$ cues $(p \mathrm{~s}<$ .017). A marginally significant Orienting Task $\times$ Cue Type $\times$ SOA interaction also emerged, $F(6,85.8)=2.03, p=.069$. Figure 2 shows the mean differences in probabilities of error between orienting tasks for each cue type and SOA. The effect of orienting task was not significant and did not significantly vary with SOA for consistent, local-neutral, or global-neutral

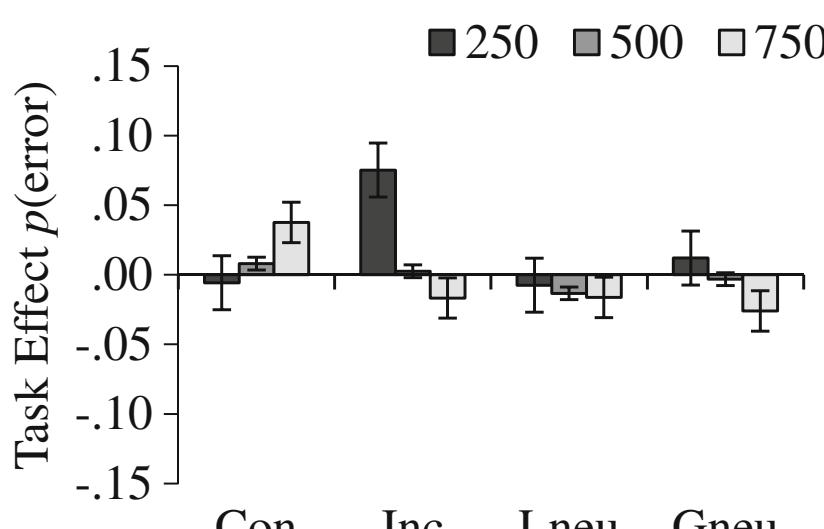

\section{Con Inc Lneu Gneu}

Fig. 2 Mean orienting task effect (local task minus global task) on probabilities of error, $p$ (error), in the secondary matching task for each cue type and SOA in Experiment 1. Positive values reflect a greater probability of error in the local orienting task, whereas negative values reflect a greater probability of error in the global orienting task. Error bars represent \pm 1 standard error cues $(p s>301)$. For inconsistent cues, the Orienting Task $\times$ SOA interaction was significant $(p=.050)$ : Whereas there was a greater probability of error in the local $(M=.259, S E=.049)$ than in the global $(M=.183, S E=.038)$ orienting task at the 250-ms SOA ( $p=.028)$, no difference between tasks was apparent at the $500-\mathrm{ms}(p=.94)$ or $750-\mathrm{ms}(p=.46)$ SOAs. This finding may reflect interference from the global level at the 250-ms SOA, which attenuated at longer SOAs. No other effects were significant $(p s>.11)$.

RTs on the primary target detection task RTs less than $100 \mathrm{~ms}$ or greater than 2.5 standard deviations above the condition means were removed (4.8\%). Data were analyzed via linear mixed modeling, with subjects and items specified as crossed random effects (Baayen, Davidson, \& Bates, 2008; Hoffman, 2014) and by-subject random slopes for withinsubjects manipulations (Barr et al., 2013). Table 1 shows the mean RTs in each condition. There were significant main effects of SOA, $F(2,66.5)=130.32, p<.001$ (targets detected faster at longer SOAs), and cue type, $F(3,89.8)=20.43, p<$ .001 (targets following local-neutral and global-neutral cues were detected faster than targets following consistent and inconsistent cues). The latter finding likely reflects a simple practice effect, given that the block of neutral-cue trials always followed the block of consistent/inconsistent-cue trials. The main effect of orienting task was marginally significant, $F(1$, $39.1)=3.28, p=.078$ (targets detected faster in the global than in the local orienting task), but this was qualified by a significant Orienting Task $\times$ SOA interaction, $F(2,64.9)=5.65, p=$ .006 (smaller effect of orienting task at longer SOAs). Regarding spatial cueing effects (Fig. 3), we observed a significant Validity $\times$ Orienting Task $\times$ Cue Type interaction, $F(4$, $54.9)=4.51, p=.001$, indicating that the effects of orienting task on the size of the cueing effects differed between cue types.

With consistent cues, we found significant cueing effects in both the local $(M=-10.83, S E=4.64, t=-2.33$, $p=.020)$ and the global $(M=-18.99, S E=4.66, t=-$ 4.07, $p<.001$ ) orienting tasks (targets were detected faster when they appeared at the location the global and local arrows pointed to), the sizes of which did not significantly differ between tasks $(M=8.16, S E=6.57, t=$ $1.24, p=.215)$. Although we did not manipulate any incentive to ignore the arrow cues, the presence of a cueing effect despite the fact that the cue was spatially uninformative is consistent with the notion that directional symbols presented at fixation induce unintentional shifts of attention to locations compatible with the meaning of the cue (e.g., Hommel et al., 2001; Pratt \& Hommel, 2003; Ristic \& Kingstone, 2006, 2012; Ristic et al., 2012).

With inconsistent cues, significant cueing effects again emerged in both the local $(M=9.50, S E=4.62, t=2.06, p$ 


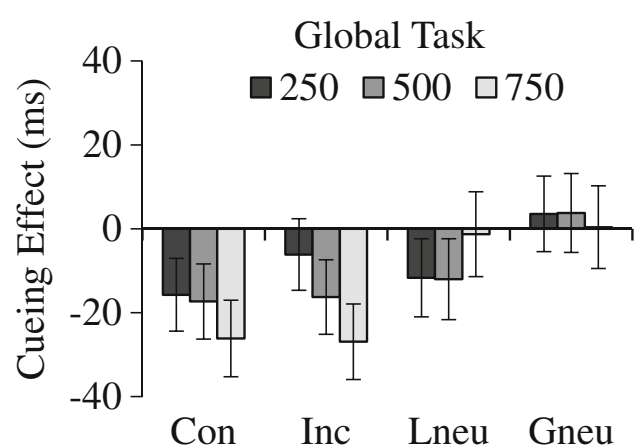

Fig. 3 Mean spatial cueing effects (valid minus invalid or, for the inconsistent cue, global-valid minus local-valid) for each cue type and SOA in the global orienting task (left) and the local orienting task (right) in Experiment 1. For the inconsistent cue, negative values reflect a cueing

$=.040)$ and the global $(M=-15.98, S E=4.69, t=-3.41, p<$ $.001)$ orienting tasks, the signs of which were in opposite directions, resulting in a significant difference between the tasks $(M=25.48, S E=6.58, t=3.87, p<.001)$. In the local orienting task, the positive sign indicates that the cueing effect was due to the local level (targets were detected faster when they appeared at the location the local arrows pointed to). In the global orienting task, the negative sign indicates that the cueing effect was due to the global level (targets were detected faster when they appeared at the location the global arrow pointed to). Thus, the orienting task determined which level of an inconsistent compound arrow cue was selected, and the arrow(s) at this level then produced an unintentional shift of attention. This pattern is consistent with a control-setting account.

With global-neutral cues, we found a significant cueing effect in the local orienting task $(M=-12.22, S E=6.19, t=$ $-1.97, p=.050$ ), with targets being detected faster when they appeared at the location the local arrows pointed to. The cueing effect in the global orienting task, however, was not significant $(M=-3.02, S E=6.47, t=-0.47, p=.641)$. Thus, when arrows were present at only the local level of a compound cue, cueing effects were observed only when the current task goal specified that the local level was task-relevant. Otherwise, these local arrows appeared to have been ignored. Since the only difference between the present global-neutral cue and the one in Mills and Dodd (2014, Exp. 3), which did produce a significant cueing effect, was the task-relevant nature of the global level, it is reasonable to interpret the present finding with global-neutral cues in terms of global precedence.

With local-neutral cues, significant cueing effects emerged once again in both the local $(M=-15.67, S E=6.12, t=-2.56$, $p=.011)$ and global $(M=-12.40, S E=6.31, t=-1.97, p=$ $.050)$ orienting tasks (targets were detected faster when they appeared at the location the global arrow pointed to), the size of which did not significantly differ between the tasks $(M=-$ $3.33, S E=9.07, t=-0.37, p=.713)$. Thus, when an arrow was

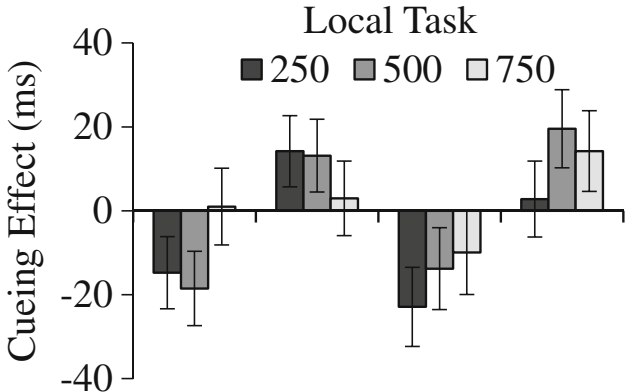

Con Inc Lneu Gneu

effect due to the global level, whereas positive values reflect a cueing effect due to the local level. Note that the global-neutral cueing effect was remapped (i.e., multiplied by -1 ) to reflect that this cueing effect was attributable to the local level. Error bars represent \pm 1 standard error

present at only the global level of a compound cue, cueing effects were observed regardless of the current task goal. This pattern speaks to the inevitability of processing global information and the optional processing of local information, in line with a global-precedence account.

The four-way interaction was not significant ( $p=.21$ ), indicating that the pattern of cueing effects did not vary with SOA. Considering that Mills and Dodd (2014) showed that the cueing effect with inconsistent cues changed with SOAsuch that a global cueing effect was observed at a $250-\mathrm{ms}$ SOA, whereas a local cueing effect was observed at a 750 $\mathrm{ms}$ SOA - the absence of an influence of SOA in the present experiment may suggest that, rather than causing the local or global advantage per se, the effect of orienting task was to enhance, attenuate, or otherwise maintain the availability of level-specific information. This would explain both the presence of a local cueing effect with inconsistent cues at the 250ms SOA, as well as the lack of an SOA effect with inconsistent cues.

To summarize, a task-compatible, level-specific cueing effect was observed when the two levels of structure conflicted (i.e., with inconsistent cues), which is in line with a controlsetting account. Yet, when there was no such conflict, the results were consistent with a global-precedence account: When the cue was a global arrow composed of local rectangles (i.e., a local-neutral cue), a cueing effect was observed regardless of orienting task, and when the cue was a global rectangle composed of local arrows (i.e., a global-neutral cue), a cueing effect was observed in the local orienting task but not in the global orienting task, findings compatible with some form of global precedence. More generally, the emerging suggestion is that shifts in precedence depend on the conflict between levels. This is consistent with Mills and Dodd (2014), with the exception that that study included no orienting task. In light of the present findings, this suggests that the level-specific advantage may not have been due to the task per se, but rather to conflict between the levels, with the effect of task serving to modulate the availability of the level- 
specific information, effectively prolonging (in the case of task-relevant global information) or expediting (in the case of task-relevant local information) the advantage at a given level.

It should be noted, however, that although the two orienting tasks clearly required responses to either the global or the local level, the arrow cues differed between the tasks, which raises the possibility that this difference may have had some influence on the results. This difference may be particularly problematic for the cues containing local arrows, because the local arrows, at the stimulus level and independent of the task, were more salient in the local orienting task (a single green arrow among red arrows) than in the global orienting task (all red arrows). By itself, this stimulus level difference-local arrows being more salient in the local than in the global orienting task - could potentially explain the finding with globalneutral cues, where a significant cueing effect was observed in the local orienting task (targets detected faster when they appeared at the location the local arrows pointed to), but not in the global orienting task. It is unclear, therefore, to what extent this finding with global-neutral cues can be attributed to the orienting task. Likewise, differential local salience between the tasks could also explain the finding with inconsistent cues, where a significant local cueing effect was observed in the local orienting task (targets detected faster when they appeared at the location the local arrows pointed to) and a significant global cueing effect was observed in the global orienting task (targets detected faster when they appeared at the location the global arrow pointed to). If local salience, independent of the orienting task, was responsible for the different patterns of cueing effects with inconsistent cues between tasks, then local salience should produce significant local cueing effects regardless of the relevancy of a particular level to a given task. In Experiment 2 we tested this possibility. In particular, Experiment 2 tested whether a local color singleton can draw attention to the local level and reverse the precedence effect.

\section{Experiment 2}

To test the possibility that local salience was responsible for the pattern of cueing effects in the local orienting task in the previous experiment, in Experiment 2, one of the local arrows was a color singleton (single green arrow among red arrows, just as in the local orienting task of Exp. 1), but there was no secondary orienting task. Accordingly, if local salience was responsible for the local cueing effects with inconsistent cues in the local orienting task in Experiment 1 (as opposed to the orienting task per se), local cueing effects should be observed once again. Moreover, just as in Experiment 1, local cueing effects with inconsistent cues should be independent of SOA. If the global orienting task was responsible for the lack of a local cueing effect with global-neutral cues in Experiment 1, then we should now find a significant local cueing effect with global-neutral cues, given that the global rectangle was no longer task-relevant.

\section{Method}

Participants Forty undergraduates from the University of Nebraska-Lincoln participated in exchange for course credit. All participants had normal or corrected-to-normal vision, were naïve to the purpose of the experiment, and were informed of their rights of participation according to the University of Nebraska-Lincoln institutional review board. None of the participants had taken part in Experiment 1.

Stimuli, procedure, and design The compound arrow cue stimuli were the same as in the local orienting task in Experiment 1. The procedure was also the same, except that there was no secondary matching task, meaning that cue processing was now entirely incidental to the detection task. Thus, participants were informed that the central arrows did not predict the location of a target and, therefore, were irrelevant to the detection task and should be ignored. The design was also the same as in Experiment 1, except that more trials were presented and that, rather than a single block of consistent/inconsistent cues being followed by one block of neutral cues, now four blocks of each were presented, which were interleaved. This modification was made to determine whether it would eliminate the suspected practice effect (i.e., the main effect of cue type) from Experiment 1. Thus, participants saw each cue (consistent, inconsistent, local-neutral, or global-neutral) at each SOA $(250,500$, or $750 \mathrm{~ms})$ and validity state (for consistent and neutral cues, valid or invalid; for inconsistent cues, global-valid or local-valid). These 24 conditions were repeated 27 times, for a total of 648 trials. The

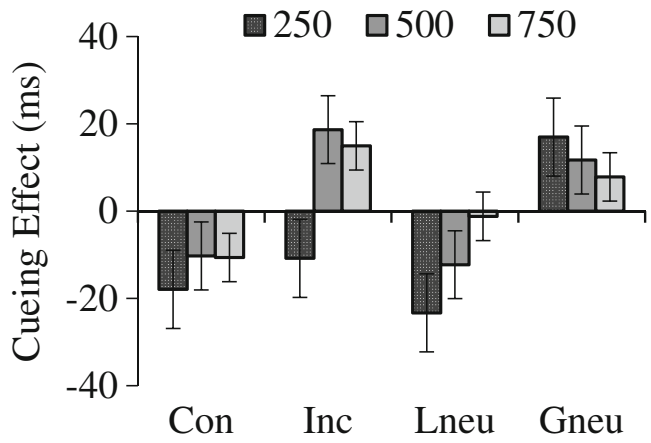

Fig. 4 Mean spatial cueing effects (valid minus invalid or, for the inconsistent cue, global-valid minus local-valid) for each cue type and SOA in Experiment 2. For the inconsistent cue, negative values reflect a cueing effect due to the global level, whereas positive values reflect a cueing effect due to the local level. Note that the global-neutral cueing effect was remapped (i.e., multiplied by -1 ) to reflect that this cueing effect was attributable to the local level. Error bars represent \pm 1 standard error 
experimental sessions took place individually and lasted $\sim 45$ min.

\section{Results and discussion}

Prior to all analyses, RTs less than $100 \mathrm{~ms}$ or greater than 2.5 standard deviations above the condition means were removed $(5.7 \%)$. The data were analyzed via linear mixed modeling, with subjects and items specified as crossed random effects (Baayen et al., 2008; Hoffman, 2014) and by-subject random slopes for within-subjects manipulations (Barr et al., 2013). We observed a significant main effect of SOA, $F(2,92.3)=$ $67.19, p<.001$ (targets detected faster at longer SOAs). The main effect of cue type, which had been significant in Experiment 1, was no longer significant, $F(3,63.9)=1.62$, $p=.19$, likely due to the increased number of blocks and their interleaving. Regarding spatial cueing effects (Fig. 4), a significant Validity $\times$ SOA interaction emerged, $F(2,66.8)=$ $4.12, p=.02$. Cueing effects were significant at the $250-\mathrm{ms}$ SOA $(M=-15.55, S E=4.69, t=-3.32, p=.002)$ and were significantly smaller at the 500-ms SOA by $(M=-12.37, S E=$ $6.11, t=-2.02, p=.05)$, as well as at the $750-\mathrm{ms}$ SOA by $(M=$ $-17.09, S E=6.17, t=-2.77, p=.007)$. The $-3.18 \mathrm{~ms}$ cueing effect at the 500-ms SOA (i.e., -15.55 minus -12.37 ) and the $1.54 \mathrm{~ms}$ cueing effect at the 750 -ms SOA (i.e., -15.55 minus $17.09)$ were not significantly different from zero $(p \mathrm{~s}>.51)$ or from each other $(t=-0.76, S E=6.18, p=.45)$. Importantly, we found a significant Cue Type $\times$ Validity interaction, $F(3$, $45.5)=4.01, p=.013$, indicating that the sizes of the cueing effects differed between cue types. Significant cueing effects occurred with consistent $(M=-13.45, S E=6.85, t=-1.96, p$ $=.05)$, local-neutral $(M=-12.76, S E=6.30, t=-2.03, p=$ $.05)$, and global-neutral $(M=-12.49, S E=6.19, t=-2.02, p=$ $.05)$ cues, none of which differed ( $p \mathrm{~s}>.92)$. Each, however, was significantly different from the cueing effect with inconsistent cues $(t \mathrm{~s}>2.78, S E \mathrm{~s} \sim 9, p \mathrm{~s}<.01)$. With inconsistent cues, the cueing effect at the 250-ms SOA was due to the global level $(M=-10.82, S E=6.31, t=-1.75, p=.078)$, but the cueing effects at the 500-ms $(M=18.67, S E=7.41, t$ $=2.52, p=.027)$ and 750-ms $(M=14.95, S E=7.63, t=1.96$, $p=.050)$ SOAs were due to the local level.

If local salience accounted for the local cueing effects in the local orienting task in Experiment 1, then local salience should produce a local cueing effect independent of the status of the local level with respect to the task. Moreover, just as in Experiment 1, the local cueing effect should not vary with SOA. The results of Experiment 2 did not support this possibility. With inconsistent cues - despite a local cueing effect at the 500-ms and 750-ms SOAs - neither a global nor a local cueing effect was found at the 250-ms SOA (in fact, the trend was toward a global cueing effect), possibly suggesting that global processing passively interfered with local processing (e.g., Navon, 1977; Sanocki, 2001) or that local salience biased the competition for selection between levels (Hommel \& Akyürek, 2009; Hommel et al., 2001; Pratt \& Hommel, 2003) by attenuating the availability of the global level and/or by enhancing the availability of the local level. In any case, these results indicate that a local-level entry point for visual processing is not obligatory, even under conditions in which it might be expected. This indicates that a local color singleton was not sufficient to produce the early and temporally stable local cueing effect with inconsistent cues in the local orienting task in Experiment 1. Similarly, with global-neutral cues, whereas no cueing effect had emerged in the global orienting task in Experiment 1, now we observed a significant and temporally stable cueing effect. This suggests that the former result was not due to the absence of a color singleton at the local level. Thus, the orienting task, not salience, appears to have been responsible for the pattern of cueing effects in Experiment 1.

\section{General discussion}

In the present study, we examined whether the influence of competing symbolic stimuli at different levels of structure on attentional control depends on top-down selection processes. Certain aspects of our results suggest that this was the case. In particular, we found positive evidence that attention could be allocated voluntarily to a specific level of a compound symbol. This was shown by a task-compatible level-specific cueing effect: With inconsistent cues in which global and local arrows pointed in opposite directions, a local cueing effect was observed in the local orienting task (target detection was faster for targets appearing at the location indicated by the local arrows), whereas a global cueing effect was observed in the global orienting task (target detection was faster for targets appearing at the location indicated by the global arrow). Thus, the current task goals determined the level of structure selected, and the directional meaning of the arrow(s) at this level subsequently induced an unintentional shift of attention. These findings are comparable with previous work that has examined the influence of competing symbols at the same level of structure (Pratt \& Hommel, 2003).

Though top-down control of attention via orienting task made it possible to select information at either level of structure depending on which level was task-relevant, other aspects of the present results suggest that this type of selection was insufficient for restricting selection only to arrows at the local level. In particular, we observed a local-neutral cueing effect (i.e., a cueing effect due to the global level) not only in the global orienting task, but also in the local orienting task, indicating that access to symbolic information at the global level was not prevented when attending to the location of a local rectangle. This finding is inconsistent with the predictions of a top-down control-setting account, which suggests that because the global arrow's directional information was irrelevant to the local 
orienting task, it should not have been processed, and therefore no cueing effect should have been observed (Folk et al., 1992). Instead, this finding is more in line with a perceptualprecedence account (Navon, 1977), which suggests that the processing of information at the global level is obligatory, whereas the processing of information at the local level is optional. According to this account, the local-neutral cueing effect was observed regardless of orienting task because there was an arrow at the highest level of globality (i.e., the relative position of an item in hierarchical space). Because processing of the global level is obligatory, this level was selected, and the arrow at this level then induced an unintentional shift of attention.

Interestingly, a global-neutral cueing effect (i.e., a cueing effect due to the local level) was observed in the local but not the global orienting task. On the one hand, this might indicate that access to symbolic information at the local level was prevented when attending to the orientation of a global rectangle, consistent with the predictions of a top-down controlsetting account. On the other hand, considering that this tasklevel compatibility effect was not observed with local-neutral cues (indicating that access to symbolic information at the global level could not be prevented when attending to the location of a local rectangle), the perceptual-precedence account seems to provide a more complete account of the data for the two neutral cue types. Accordingly, the global-neutral cueing effect may reflect the processing of information at the local level being optional (Navon, 1977). That is, because the local level was not task-relevant in the global orienting task, it was not processed, and as a result no cueing effect was observed. Taken together, the patterns of spatial cueing effects for the two types of neutral cues suggest that cueing effects triggered by the global level may be more strongly reflexive than those triggered by the local level, insofar as the spatial orienting effect for local-neutral cues (due to global level) could withstand violations to a nonspatial task-level attentional set, whereas the spatial orienting effect for the globalneutral cue (due to the local level) could not.

Although the two orienting tasks clearly required responses to either the global or the local level, the natures of the tasks differed, which raises the possibility that this difference may have had some influence on the results. Namely, the local and global orienting tasks differed not only in terms of whether a response to either the local or the global level of a compound cue was required, but also in terms of the salience of the arrows at the local level. Therefore, the local cueing effect with inconsistent cues in the local orienting task may have been driven by salience as opposed to orienting task. Similarly, the null local cueing effect with global-neutral cues in the global orienting task may have been due to a nonsalient local level. In Experiment 2 we examined this possibility. This experiment was a replication of Mills and Dodd (2014), with the exception that one of the local arrows was a color singleton. As in that study, with inconsistent cues, we found a global cueing effect at the 250-ms SOA and local cueing effects at the longer SOAs. Thus, with inconsistent cues, a color singleton at the local level did not produce early local cueing effects (Exp. 2) unless the local level was task-relevant (Exp. 1). Likewise, with global-neutral cues, we found significant cueing effects regardless of SOA. These findings suggest that the effects of orienting task on the sizes of the cueing effects with inconsistent and global-neutral cues in Experiment 1 were indeed due to orienting task, not salience. Accordingly, we conclude that the results support a version of global precedence in which the task set can either maintain the availability of the global level (evident by the absence of SOA effects on global cueing effects when the global level was task-relevant; Exp. 1) or instead attenuate its availability (evident by the absence of SOA effects on local cueing effects when the local level was task-relevant; Exp. 1).

According to Navon's $(1977,2003)$ global-precedence hypothesis, the availability of the global level is constant and does not attenuate, even when attention is focused on the local level. Subsequent findings, however, have suggested that this assumption may be too strong, since there is ample evidence that the availabilities of different levels of structure of hierarchical patterns vary with time (e.g., Kimchi, 1998; Mills \& Dodd, 2014). An effect of processing duration (i.e., SOA) on the global advantage (Exp. 2; see also Mills \& Dodd, 2014), therefore, does not support Navon's original globalprecedence hypothesis, but it is consistent with a weaker version in which the availability of the global level can be modulated over time (Navon, 1991). Therefore, we speculate that an effect of SOA was not observed in Experiment 1 because the task relevancy of a particular level modulated the availability of that level, effectively prolonging (in the case of task-relevant global information) or expediting (in the case of task-relevant local information) the availability, and thus the dominance, of level-specific information. Only in the absence of direct task demands is there an obligatory global-to-local change in advantage (Exp. 2; Mills \& Dodd, 2014), and even then, only in the presence of conflict. The emerging picture here is that shifts in precedence depend on conflict between levels.

So far, experiments using the compound-arrow cueing procedure have required only simple detection responses to a $1^{\circ}$ target (Exps. 1-2; see also Mills \& Dodd, 2014). It is possible that such a simple (coarse) judgment might have permitted what Theeuwes (2004) has referred to as a "wide attentional window." If detection responses to a $1^{\circ}$ target permitted a wide attentional window, then the spatial cueing task may have introduced a top-down bias toward global processing across all conditions. This bias presumably would have an additive effect with the orienting bias manipulated with the secondary matching task in Experiment 1 and, therefore, may be overpowered by conditions conspiring to produce a local advantage. This overall top-down bias toward processing the global level, speculative as it may be, might have manifested 
itself in the finding that when the cue was a global arrow composed of local rectangles (local-neutral cue) a cueing effect was observed even in the local orienting task (Exp. 1, Fig. 3). Although this hypothesis is attractive, at least three pieces of evidence cast doubt on it. First, we obtained a (global) cueing effect with local-neutral cues in the local orienting task. Since previous work has shown that top-down attention to pop-out local items weakens the global advantage (Han \& He, 2003), it might be expected that a top-down bias toward global processing induced by a wide attentional window should be weakened in the local orienting task and, therefore, that the local-neutral cueing effect should be smaller than in the global orienting task. However, we found no difference between the tasks - if anything, the effect was larger in the local orienting task. Second, Lamb and Robertson (1988) found that responses to the local level of a central hierarchical stimulus were longer when stimuli occurred randomly and were mixed with peripherally presented stimuli, as compared with a condition in which all stimuli were always presented at central fixation. They suggested that with central presentation, a smaller focus of attention could be maintained, leading to enhanced local processing. Because our cues were presented at central fixation, it might be expected that no global advantage should result, given that attention was consistently directed at a level (via the task manipulation) within a block of trials. Finally, we considered something like this hypothesis in Mills and Dodd (2014, Exp. 3), in which cue-target eccentricity was manipulated, the logic being that a wide attentional window would predict a cue-target proximity effect. However, none was found-eccentricity did not alter the pattern. Consequently, spatial uncertainty, presumably a condition of diffuse or wide attention, does not seem to affect the size of the global advantage, at least not when cue processing is incidental and both levels are related to as objects.

In summary, we found that interactions between the task demands and the structure of the input information selectively modulate the relative needs of visual information at different levels of stimulus structure. To select symbols at different levels of structure, therefore, the present work suggests that at least two factors need to be considered: the observer's current task, which specifies the demands of visual information from the input, and the globality of this visual information across the percept, which specifies the availability of the percept for selection. Studies investigating the determinants of selection efficiency typically draw on tasks requiring selective attention to centrally presented local information while ignoring irrelevant peripheral information, as in flanker tasks. Moreover, the stimuli used in these studies are generally perceptually simple, containing semantic information at only one level of stimulus structure, as in a single central arrow. Many structural differences exist between the stimuli presented in traditional studies of selectivity and the sort of stimuli found in the real world (e.g., hierarchical levels of structure, depth planes). Considering that differences in stimulus structure are known to influence processing strategies (Garner, 1970, 1974), understanding the role of stimulus structure in information processing will be crucial for understanding how various factors influence selective attention. Ignoring the nature of the stimulus would lead, at worst, to incorrect assessment of the nature of information processing, and, at best, to an inadequate picture. A goal for future research, therefore, should be to understand how other dimensions of stimulus structure, such as a target's location in depth on a three-dimensional plane, contribute to selectivity.

Author note We thank Howard Egeth for his insight and guidance throughout the development of the manuscript. We also thank Brett Bahle and three anonymous reviewers.

\section{References}

Baayen, R. H., Davidson, D. J., \& Bates, D. M. (2008). Mixed-effects modeling with crossed random effects for subjects and items. Journal of Memory and Language, 59, 390-412. doi:10.1016/j. jml.2007.12.005

Barr, D. J., Levy, R., Scheepers, C., \& Tily, H. J. (2013). Random effects structure for confirmatory hypothesis testing: Keep it maximal. Journal of Memory and Language, 68, 255-278. doi:10.1016/j. jml.2012.11.001

Chasteen, A. L., Burdzy, D. C., \& Pratt, J. (2010). Thinking of god moves attention. Neuropsychologia, 48, 627-630.

Corbetta, M., \& Shulman, G. L. (2002). Control of goal-directed and stimulus-driven attention in the brain. Nature Reviews Neuroscience, 3, 201-215.

Desimone, R., \& Duncan, J. (1995). Neural mechanisms of selective visual attention. Annual Review of Neuroscience, 18, 193-222. doi:10.1146/annurev.ne.18.030195.001205

Dodd, M. D., Van der Stigchel, S., Leghari, M. A., Fung, G., \& Kingstone, A. (2008). Attentional SNARC: There's something special about numbers (let us count the ways). Cognition, 108, 810-818.

Egeth, H. E., \& Yantis, S. (1997). Visual attention: Control, representation, and time course. Annual Review of Psychology, 48, 269-297. doi:10.1146/annurev.psych.48.1.269

Enns, J. T., Austen, E. L., Di Lollo, V., Rauschenberger, R., \& Yantis, S. (2001). New objects dominate luminance transients in setting attentional priority. Journal of Experimental Psychology: Human Perception and Performance, 27, 1287-1302. doi:10.1037/00961523.27.6.1287

Estes, Z., Verges, M., \& Barsalou, L. W. (2008). Head up, foot down: Object words orient attention to the objects' typical location. Psychological Science, 19, 93-97.

Fattorini, E., Pinto, M., Rotondaro, F., \& Doricchi, F. (2015). Perceiving numbers does not cause automatic shifts of spatial attention. Cortex, 73, 298-316.

Fischer, M. H., Castel, A. D., Dodd, M. D., \& Pratt, J. (2003). Perceiving numbers causes spatial shifts of attention. Nature Neuroscience, 6 , $555-556$.

Folk, C. L., \& Remington, R. (1998). Selectivity in distraction by irrelevant featural singletons: Evidence for two forms of attentional capture. Journal of Experimental Psychology: Human Perception and Performance, 24, 847-858. doi:10.1037/0096-1523.24.3.847

Folk, C. L., Remington, R. W., \& Johnston, J. C. (1992). Involuntary covert orienting is contingent on attentional control settings. 
Journal of Experimental Psychology: Human Perception and Performance, 18, 1030-1044. doi:10.1037/0096-1523.18.4.1030

Garner, W. R. (1970). The stimulus in information processing. American Psychologist, 25, 350.

Garner, W. R. (1974). The processing of information and structure. Potomac, MD: Erlbaum.

Han, S., \& He, X. (2003). Modulation of neural activities by enhanced local selection in the processing of compound stimuli. Human Brain Mapping, 19, 273-281.

Hoffman, L. (2014). Longitudinal analysis: Modeling within-person fluctuation and change. New York, NY: Routledge.

Hommel, B., \& Akyürek, E. G. (2009). Symbolic control of attention: Tracking its temporal dynamics. Attention, Perception, \& Psychophysics, 71, 385-391. doi:10.3758/APP.71.2.385

Hommel, B., Pratt, J., Colzato, L., \& Godijn, R. (2001). Symbolic control of visual attention. Psychological Science, 12, 360-365.

Itti, L., \& Koch, C. (2000). A saliency-based search mechanism for overt and covert shift of visual attention. Vision Research, 40, 1489-1506. doi:10.1016/S0042-6989(99)00163-7

Jonides, J. (1981). Voluntary versus automatic control over the mind's eye's movement. In J. Long \& A. Baddeley (Eds.), Attention and performance IX (pp. 187-203). Hillsdale, NJ: Erlbaum.

Kimchi, R. (1998). Uniform connectedness and grouping in the perceptual organization of hierarchical patterns. Journal of Experimental Psychology: Human Perception and Performance, 24, 1105-1118. doi:10.1037/0096-1523.24.4.1105

Lamb, M. R., \& Robertson, L. (1988). The processing of hierarchical stimuli: Effects of retinal locus, location uncertainty, and stimulus identity. Perception \& Psychophysics, 44, 172-181.

Mills, M., \& Dodd, M. D. (2014). Which way is which? Examining global/local processing with symbolic cues. Journal of Experimental Psychology: Human Perception and Performance, $143,1429-1436$

Mills, M., Smith, K. B., Hibbing, J. R., \& Dodd, M. D. (2015). Obama cares about visuo-spatial attention: Perception of political figures moves attention and determines gaze direction. Behavioural Brain Research, 278, 221-225.

Navon, D. (1977). Forest before trees: The precedence of global features in visual perception. Cognitive Psychology, 9, 353-383. doi:10. 1016/0010-0285(77)90012-3

Navon, D. (1991). Testing a queue hypothesis for the processing of global and local information. Journal of Experimental Psychology: General, 120, 173-189. doi:10.1037/0096-3445.120.2.173
Navon, D. (2003). What does a compound letter tell the psychologist's mind? Acta Psychologica, 114, 273-309.

Posner, M. I. (1980). Orienting of attention. Quarterly Journal of Experimental Psychology, 32, 3-25. doi:10.1080/ 00335558008248231

Pratt, J., \& Hommel, B. (2003). Symbolic control of visual attention: The role of working memory and attentional control settings. Journal of Experimental Psychology: Human Perception and Performance, 29, 835-845. doi:10.1037/0096-1523.29.5.835

Remington, R., Johnston, J. C., \& Yantis, S. (1986). Do abrupt onsets capture attention involuntarily? Bulletin of the Psychonomic Society, 24, 347.

Ristic, J., \& Kingstone, A. (2006). Attention to arrows: Pointing to a new direction. Quarterly Journal of Experimental Psychology, 59, 19211930. doi:10.1080/17470210500416367

Ristic, J., \& Kingstone, A. (2012). A new form of human spatial attention: Automated symbolic orienting. Visual Cognition, 20, 244-264. doi: 10.1080/13506285.2012.658101

Ristic, J., Landry, M., \& Kingstone, A. (2012). Automated symbolic orienting: The missing link. Frontiers in Psychology, 3, 1-7.

Sanocki, T. (2001). Interaction of scale and time during object identification. Journal of Experimental Psychology: Human Perception and Performance, 27, 290-302.

Theeuwes, J. (1990). Perceptual selectivity is task-dependent: Evidence from selective search. Acta Psychologica, 74, 81-99. doi:10.1016/ 0001-6918(90)90036-F

Theeuwes, J. (1994). Stimulus-driven capture and attentional set: Selective search for color and visual abrupt onsets. Journal of Experimental Psychology: Human Perception and Performance, 20, 799-806. doi:10.1037/0096-1523.20.4.799

Theeuwes, J. (2004). Top-down search strategies cannot override attentional capture. Psychonomic Bulletin \& Review, 11, 65-70. doi:10. 3758/BF03206462

Weger, U. W., \& Pratt, J. (2008). Time flies like an arrow: Space-time compatibility effects suggest the use of a mental timeline. Psychonomic Bulletin \& Review, 15, 426-430. doi:10.3758/PBR. 15.2 .426

Yantis, S. (2000). Goal directed and stimulus driven determinants of attentional control. In S. Monsell \& J. Driver (Eds.), Attention and performance XVIII (pp. 73-104). Cambridge, MA: MIT Press.

Yantis, S., \& Jonides, J. (1984). Abrupt visual onsets and selective attention: Evidence from visual search. Journal of Experimental Psychology: Human Perception and Performance, 10, 601-621. doi:10.1037/0096-1523.10.5.601 